\title{
Reduced-Order Unscented Kalman Filter for Sensorless Control of Permanent-Magnet Synchronous Motor
}

\author{
Cheol Moon* and Young Ahn Kwon ${ }^{\dagger}$
}

\begin{abstract}
The unscented Kalman filter features a direct transforming process involving unscented transformation for removing the linearization process error that may occur in the extended Kalman filter. This paper proposes a reduced-order unscented Kalman filter for the sensorless control of a permanent magnet synchronous motor. The proposed method can reduce the computational load without degrading the accuracy compared to the conventional Kalman filters. Moreover, the proposed method can directly estimate the electrical rotor position and speed without a back-electromotive force. The proposed Kalman filter for the sensorless control of a permanent magnet synchronous motor is verified through the simulation and experimentation. The performance of the proposed method is evaluated over a wide range of operations, such as forward and reverse rotations in low and high speeds including the detuning parameters.
\end{abstract}

Keywords: Unscented Kalman filter, Reduced order model, Unscented transformation, Sensorless speed control, Permanent-magnet synchronous motor

\section{Introduction}

Many studies on the vector control of permanent-magnet synchronous motor (PMSM) drives without position sensors have been reported [1]. Sensorless control using an extended Kalman filter (EKF) facilitates the best estimation of state values, regardless of system and sensor noise [2]. However, the linearization process of this EKF often involves a difference between the modified model and the original system model.

Julier and Uhlman presented a new type of Kalman filter called the unscented Kalman filter (UKF), which employs unscented transformation (UT) [3-4]. Unlike the EKF, which has a linearization process method, the UKF features a direct mapping process involving UT, without a modified system model. Despite these advantages, the algorithm of such Kalman filters has a heavy computational load, which is a major drawback. Therefore, it is necessary to minimize the state variables in order to reduce the computational time.

The sensorless control of a PMSM with a reduced-order Kalman filter was previously reported [5-8]. A thirdorder EKF with a back-electromotive force for interior PMSM sensorless control was proposed in [5]. A thirdorder linear Kalman filter was also proposed; however, its implementation is difficult [6]. Another study introduced a parallel reduced-order EKF with a second-order parallel form at a low speed and a third-order parallel form at a high speed [7]. Recently, a square-root EKF with state

$\dagger$ Corresponding Author: Dept. of Electrical and Computer Engineering, Pusan National University, Korea. (yakwon@pusan.ac.kr)

* Dept. of Electrical and Computer Engineering, Pusan National

University, Korea. (mch99100@pusan.or.kr)

Received: February 7, 2016; Accepted: October 28, 2016 and output variables in the form of a $2 \times 1$ matrix was introduced [8]. However, with the previously reported paper of the Kaman filter using reduced form, an additional algorithm is needed to estimate the electrical-rotor position and speed, and the state estimation values are inaccurate in the low-speed region (lower than 10\% of the rated speed). And practically no research has been conducted to reduce the order of UKF algorithms in this motors control field.

The type of UT depends on the scaling-parameter values [9-10]. However, control is difficult to apply owing to the limited information about the scaling-parameter values, how the observer is affected by the scaling parameters, and how the scaling parameters should be selected. Various types of UT, such as basic UT, general UT, and scaled UT can be specified according to the distribution of sigma points and weights.

Experimental results for basic UT ( $\alpha=1, \beta=0, \kappa=0)$ and general UT ( $\alpha=1, \beta=0, \kappa=0.5,1,36)$ based on scaling parameters have been presented [10]. A previous work summarizes the scaling parameters and explains how to select and perform a simple comparison simulation of basic UT ( $\alpha=1, \beta=0, \kappa=0)$, general UT $(\alpha=1, \beta=0, \kappa=$ $0.1-0.9$ ). Several experimental results for various types of UT, such as basic UT and general UT $(\kappa=1)$, have been presented [11]. The application of a UKF with basic UT having the parameter $\beta(\alpha=1, \beta=2, \kappa=0)$ was reported [12].

This paper investigates the application, design, and implementation of a reduced-order unscented Kalman filter (ROUKF) for state estimation of the motor drive without a position sensor. Experimental results for a wide range of operations, such as forward and reverse rotation at low and rated mechanical speeds; detuning parameters; 
and load tests demonstrated the quality of the estimation performance.

\section{Reduced-order Model of Permanet Magnet Synchronous Motor}

Motor mathematical modeling using the $\alpha \beta$ stationary reference frame serves to reduce the complexity of the three-phase voltage equation. If the change in the electrical speed is assumed to be constant for one sampling period, the differential value is considered to be zero. The general full-order form is as follows [1].

$$
\frac{d}{d t}\left[\begin{array}{c}
i_{\alpha s} \\
i_{\beta s} \\
\omega_{r} \\
\theta_{r}
\end{array}\right]=\frac{1}{L_{s}}\left[\begin{array}{c}
V_{\alpha s}-R_{s} i_{\alpha s}+\lambda_{m} \omega_{r} \sin \left(\theta_{r}\right) \\
V_{\beta S}-R_{s} i_{\beta s}-\lambda_{m} \omega_{r} \cos \left(\theta_{r}\right) \\
0 \\
L_{s} \omega_{r}
\end{array}\right]
$$

Here, $V_{\alpha \beta s}$ are the $\alpha \beta$ axis stator voltages, $i_{\alpha \beta s}$ are the $\alpha \beta$ axis stator currents, $L_{s}$ is the stator inductance, and $R_{s}$ is the stator resistance. $\lambda_{m}$ is the flux linkage from the permanent magnet, $\omega_{r}$ is the electrical speed, and $\theta_{r}$ is the electricalrotor position.

The state matrix of the reduced-order form is a $3 \times 1$ matrix, and the output matrix is a $2 \times 1$ matrix. The state matrix of the reduced-order model is $\mathbf{x}=\left[\begin{array}{lll}i_{\alpha s} & \omega_{r} & \theta_{r}\end{array}\right]^{T}$, and the output matrix is $\mathbf{y}=\left[\begin{array}{ll}i_{\alpha s} & i_{\beta s}\end{array}\right]^{T}$. The reduced-order discrete forms are as follows.

$$
\begin{gathered}
x_{1(k)}=a x_{1(k-1)}+b x_{2(k-1)} \sin \left(x_{3(k-1)}\right)+c u_{1(k-1)} \\
x_{2(k)}=x_{2(k-1)} \\
x_{3(k)}=x_{3(k-1)}+\Delta t x_{2(k-1)} \\
y_{1(k)}=x_{1(k)} \\
y_{2(k)}=a y_{2(k-1)}+b x_{2(k-1)} \sin \left(x_{3(k-1)}\right)+c u_{2(k-1)}
\end{gathered}
$$

Here, $\Delta t$ is the sampling time, $a=\left(1-\Delta t R_{S} / L_{S}\right), b=\Delta t \lambda_{m} /$ $L_{S}$, and $c=\Delta t L_{S}$.

\section{Unscented Kalman Filter}

Compared with the EKF, the UKF has the same structure, including the prediction, Kalman gain, and correction step. The only difference is the UT method instead of the linearization process. Moreover, unlike Monte Carlo simulation, the proper number of samples for state estimation can be determined according to the state variables. The purpose of the UT method is to generate sigma points $\chi$ and weights $\omega$ using mean and covariance values of the function $f(x)$. Such a UT is affected by the scaling parameters $\alpha, \beta, \kappa$, and $\lambda$ which specify the distribution of sigma points and weights.
In scaled UT $[3,4], \alpha, \beta$, and $\kappa$ are nonzero, and $\alpha$ is less than 1 . Generally, the sum of the weights equals 1 , except in the case of scaled UT, because of the $\beta$ parameter, which is used to create a different weight $W_{0}$ for an error covariance $y$. The sigma points and weights are defined as follows $[3,4]$.

First, the $\lambda$ scaling parameter value is selected.

Second, the upper triangular matrix $\mathbf{u}_{i}$ is calculated using Cholesky factorization.

Third, the sigma points and weights are determined.

$$
\begin{array}{ll}
\lambda=\alpha^{2} \kappa+\left(1-\alpha^{2}\right) n \\
& \mathbf{U}^{T} \mathbf{U}=(n+\lambda) \mathbf{P}_{x} \\
\boldsymbol{\chi}_{0}=\mathbf{x}, & W_{0}=\lambda /(n+\lambda) \\
& W_{0}^{\left[P_{y}\right]}=W_{0}+1-\alpha^{2}+\beta, \\
\boldsymbol{\chi}_{i}=\mathbf{x}+\mathbf{u}_{i}, & W_{i}=1 / 2(n+\lambda), \\
\boldsymbol{\chi}_{i+n}=\mathbf{x}-\mathbf{u}_{i} & W_{i+n}=W_{i}, i=1,2, \ldots, n .
\end{array}
$$

Here, $n$ is the number of state variables.

The case where $\lambda=0(\alpha=1$ and $\kappa=0)$ and $\beta=0$ is called basic UT [10]. The $0^{\text {th }}$ sigma point $\chi_{0}$ and weight $W_{0}$ are removed, and the sum of the weights is 1 . The number of state variables is $n$, and the number of sigma points is $2 n^{2}$. The case where $\alpha=1$ and $\beta=0$, is called general UT. If $\kappa$ equals zero point five $(\kappa=0.5)$, the general UT [10] has the same weight values. The number of sigma points is $2 n^{2}+n$, and the sum of the weights is 1 .

The UKF but for the UT has three steps: prediction, Kalman gain, and estimation. This procedure is as follows $[3,4]$.

Initialization;

Calculation of sigma points and weights;

Prediction of the estimation value and error covariance;

Prediction of the measurement value and error covariance;

Calculation of the Kalman gain;

Correction of the estimation state and covariance.

$$
\begin{gathered}
\hat{\mathbf{x}}_{k \mid k-1}=\sum_{i=0}^{2 n} W_{i} \mathbf{f}\left(\boldsymbol{\chi}_{i}\right) . \\
\mathbf{P}_{x, k \mid k-1}=\sum_{i=0}^{2 n} W_{i}\left(\mathbf{f}\left(\boldsymbol{\chi}_{i}\right)-\hat{\mathbf{x}}_{k \mid k-1}\right)\left(\mathbf{f}\left(\boldsymbol{\chi}_{i}\right)-\hat{\mathbf{x}}_{k \mid k-1}\right)^{T}+\mathbf{Q} . \\
\hat{\mathbf{y}}_{k \mid k-1}=\sum_{i=0}^{2 n} W_{i} \mathbf{h}\left(\boldsymbol{\chi}_{i}\right) . \\
\mathbf{P}_{y, k \mid k-1}=\sum_{i=0}^{2 n} W_{i}\left(\mathbf{h}\left(\boldsymbol{\chi}_{i}\right)-\hat{\mathbf{y}}_{k \mid k-1}\right)\left(\mathbf{h}\left(\boldsymbol{\chi}_{i}\right)-\hat{\mathbf{y}}_{k \mid k-1}\right)^{\mathrm{T}}+\mathbf{R} . \\
\mathbf{P}_{x y, k \mid k-1}=\sum_{i=0}^{2 n} W_{i}\left(\mathbf{f}\left(\boldsymbol{\chi}_{i}\right)-\hat{\mathbf{x}}_{k \mid k-1}\right)\left(\mathbf{h}\left(\boldsymbol{\chi}_{i}\right)-\hat{\mathbf{y}}_{k \mid k-1}\right)^{\mathrm{T}} . \\
\mathbf{K}_{k}=\mathbf{P}_{\mathrm{xy}} \mathbf{P}_{y}^{-1} . \\
\hat{\mathbf{x}}_{k}=\hat{\mathbf{x}}_{k \mid k-1}+\mathbf{K}_{\mathrm{k}}\left(\mathbf{y}_{k}-\hat{\mathbf{y}}_{\mathrm{k}}\right) . \\
\mathbf{P}_{k}=\mathbf{P}_{k \mid k-1}-\mathbf{K}_{\mathrm{k}} \mathbf{P}_{y} \mathbf{K}_{k}^{T} .
\end{gathered}
$$




\section{Simulation}

To evaluate the performance of the proposed method with the UT, a computer simulation model was constructed using the language $\mathrm{C}$. The stator resistance and inductance values of a PMSM (eight-pole $750-\mathrm{W}$ class) are $1.5 \Omega$ and $4.87 \mathrm{mH}$. And the rated speed and torque of this motor are 2,000 rpm and $3.58 \mathrm{Nm}$.

The overall block diagram for a PMSM drive without a position sensor for vector control is shown in Fig. 1. This motor model is analyzed in the simulation by using the

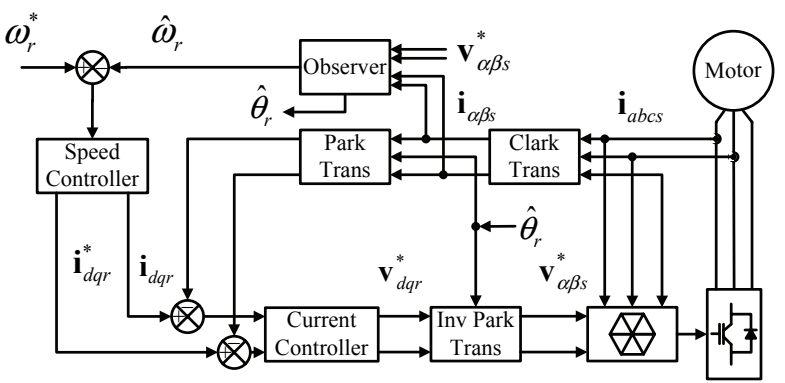

Fig. 1. Vector-control PMSM drive without a position sensor.

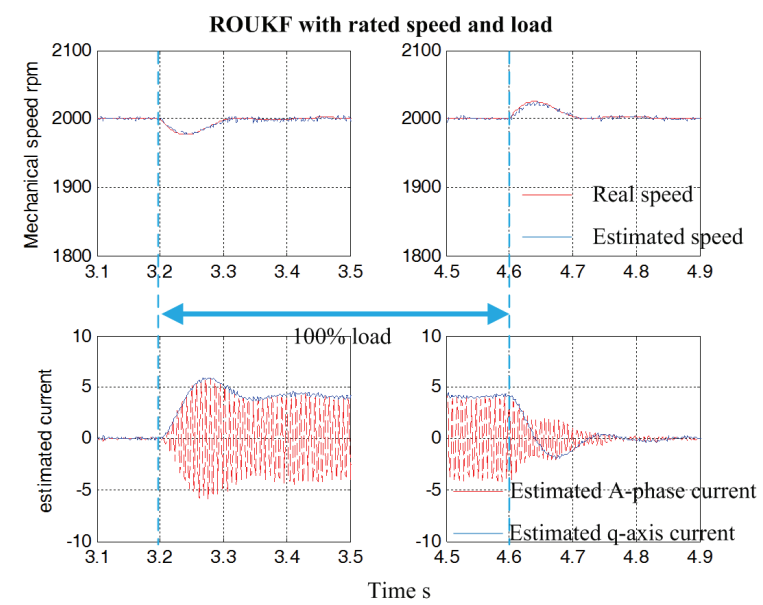

(a)

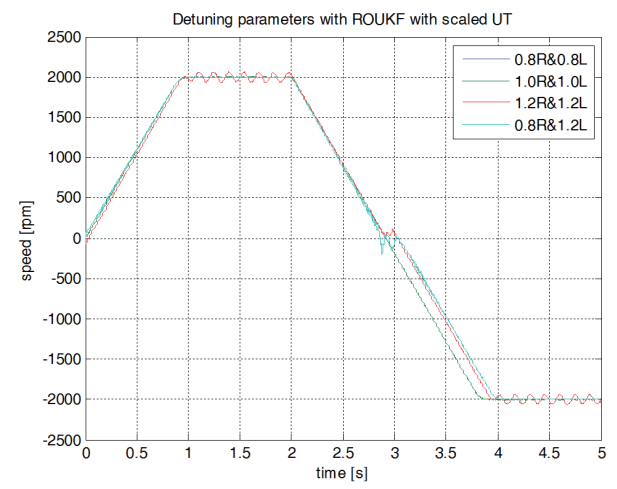

(b)

Fig. 2. Simulation results for the proposed method: (a) load test $(500 \mathrm{rpm}, \quad 0 \rightarrow 100 \rightarrow 0 \%$ torque load), (b) detuning-parameter test $(2000 \rightarrow-2000 \mathrm{rpm})$ fourth-order Runge-Kutta method (numerical analysis) at intervals of $1 \mu \mathrm{s}$. The current and speed sampling time are $200 \mu \mathrm{s}$ and $1 \mathrm{~ms}$, respectively. The switching frequency is $2.5 \mathrm{kHz}$, according to the symmetric space vector pulsewidth modulation method.

Fig. 2 shows the simulation results for the motor-drive algorithm without position sensors using the proposed reduced-order form with scaled UT ( $\alpha=1, \beta=0.1, \kappa=0.5)$. Each figure presents the performance evaluation for the estimated mechanical speed. Fig. 2(a) shows the results of a rated-load test $(3.58 \mathrm{Nm})$, which indicate that the proposed scheme gives satisfactory results. Fig. 2(b) presents the speed response according to each detuningparameter value according to the rated reference speed $(2,000 \rightarrow-2,000 \mathrm{rpm})$. If the detuning stator resistance is far lower than the real value, the estimated speed near the zero point is unclear. And if the detuning stator inductance is far higher than the real value, the estimated speed oscillates during the steady state.

\section{Experimental Result and Discussion}

Fig. 3 shows the experimental equipment used to analyze an eight-pole 1-horsepower (HP) PMSM. A Texas Instruments $150-\mathrm{MHz}$ TMS320F28335 module was used for the main arithmetic unit. The PMSM was equipped with a 2000 PPR incremental encoder to measure the actual speed and position.

To verify the performance of the proposed method, the full-order form with basic UT $(\alpha=1, \beta=0, \kappa=0)$ and the reduced-order form with scaled UT $(\alpha=1, \beta=0.1, \kappa=0.5)$ were compared.

Fig. 4 shows experimental waveforms of the forward and reverse rotations at a high speed (2,000 to $-2,000 \mathrm{rpm}$, $100 \%$ of rated speed). Figs. 4(a) and (b) show experimental results for the full and reduced-order forms. The figures sequentially present the real electrical-rotor position (blue line), the estimated electrical-rotor position (green line), the error (orange line) during the initial transient state, the real mechanical speed (yellow line), the estimated mechanical speed (red line), and the error between the real and estimated mechanical speeds (orange line). The motor runs at zero speed, increases to 2,000 rpm for $2 \mathrm{~s}$, and then decreases to $-2,000 \mathrm{rpm}$. In the case of the full-order form, this indicates that the initial rising time is less than $150 \mathrm{~ms}$ and means that the speed response is $13.333 \mathrm{rpm}$ per $1 \mathrm{~ms}$

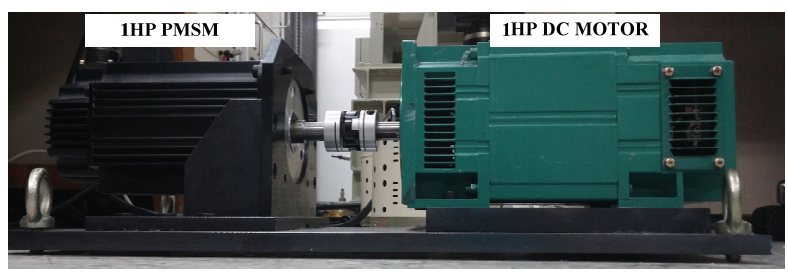

Fig. 3. 1-HP PMSM with load. 

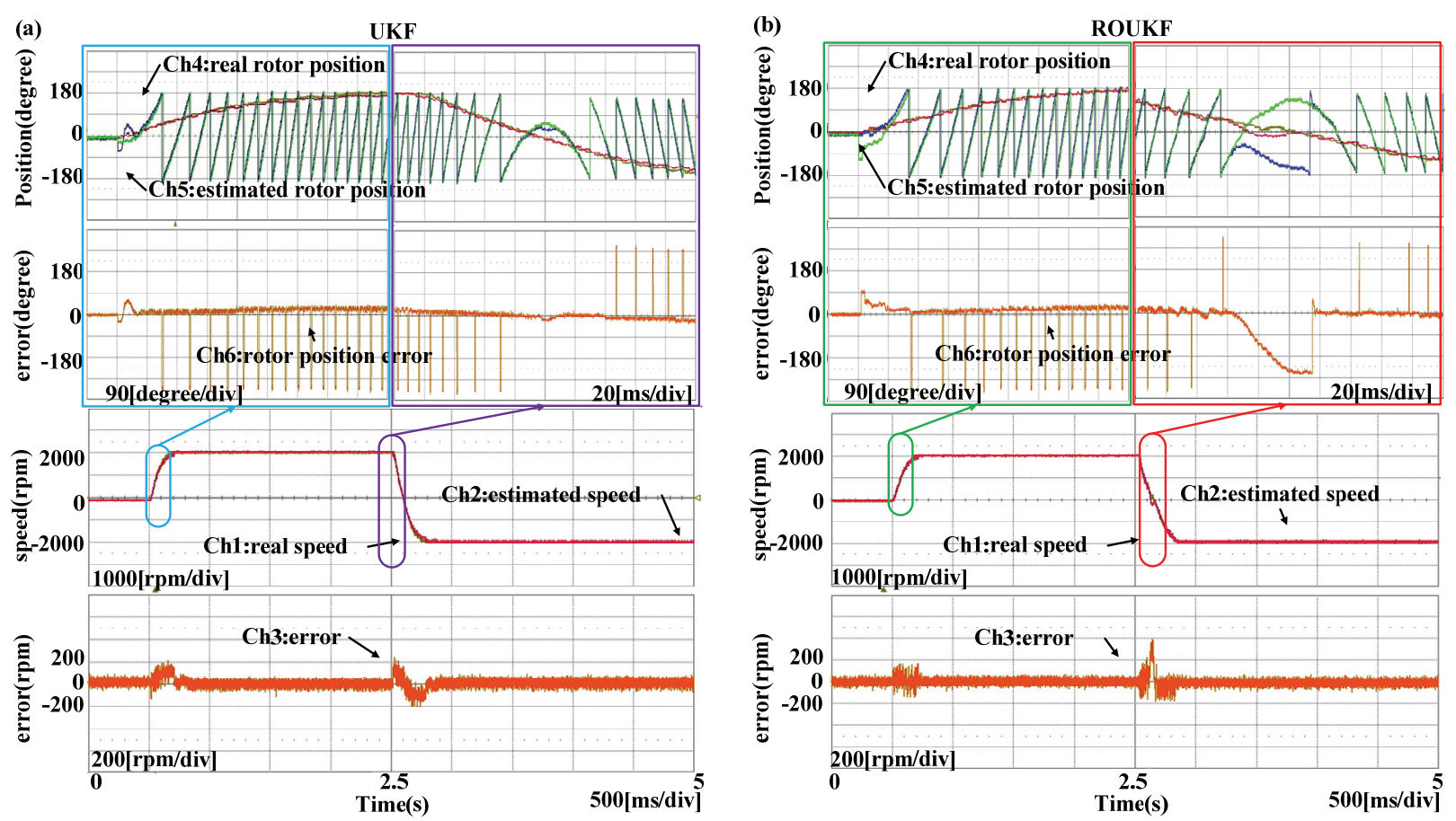

Fig. 4. Experimental results for the rated forward and reverse rotations $(2,000 \rightarrow-2,000 \mathrm{rpm})$ : (a) full-order form; (b) reduced-order form
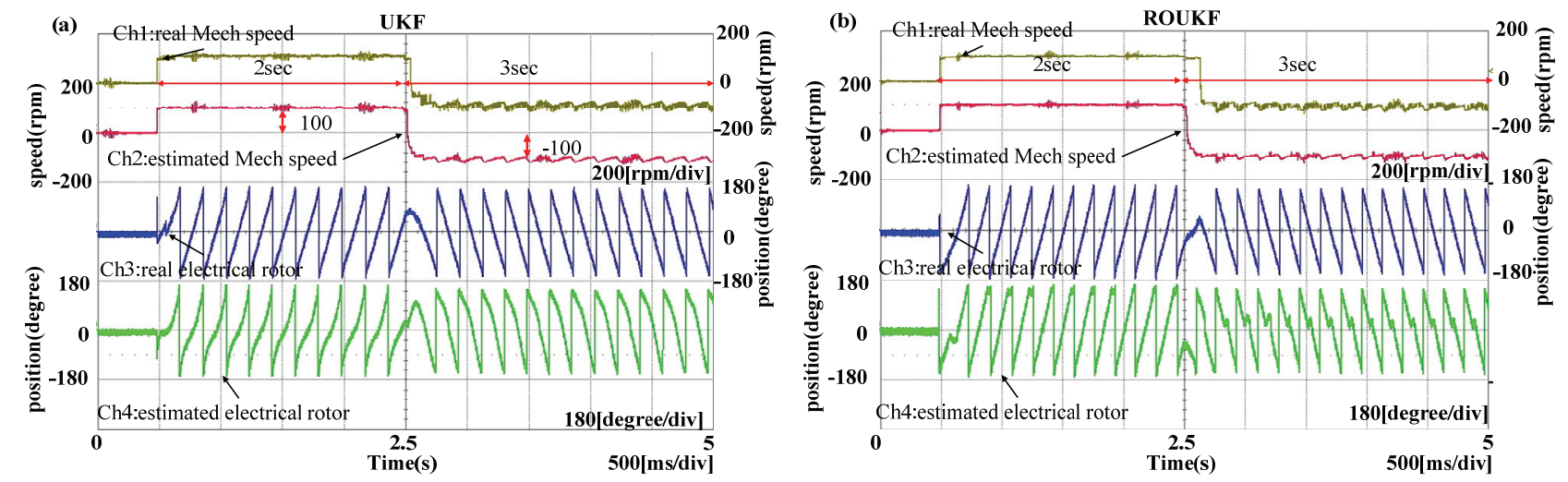

Fig. 5. Real and estimated electrical position and mechanical speed at low speeds $(100 \rightarrow-100 \mathrm{rpm}, 5 \%$ of rated speed): (a) full-order form; (b) reduced-order form

(here, $1 \mathrm{~ms}$ is the speed controller sampling time). For the reverse rotation, Figures show the exact speed estimation near the zero point. In the case of the reduced-order form, this method performs well in the initial transient and steady state. However, it does not meet performance standards near the zero point in a moment ( $40 \mathrm{~ms})$. The appropriate system model variable values $\mathbf{Q}$ and $\mathbf{R}$ should have been determined according to the new reduced-order model.

Fig. 5 presents the forward and reverse rotations at low speeds (100 to $-100 \mathrm{rpm}, 5 \%$ of rated speed). As shown in the experimental results, the full-order form performed relatively well. The proposed method presents some differences between the actual and estimated mechanical speeds in the reverse transient state; however, they converge quickly. The estimated electrical-rotor position experiences a slight overshoot near the zero point. This indicates that the proposed method has proper estimation and control performance for low speed.

To ensure robust performance, a load experiment was conducted. Fig. 6 shows the experimental results obtained with $2.7 \mathrm{Nm}$ (75\% of the rated load) applied to the PMSM and removed after $1.6 \mathrm{~s}$ at $500 \mathrm{rpm}$. This method has a slightly inaccurate estimated value when a load is applied. This estimated state affects the covariance value, and as a result, the performance of the other estimated values deteriorates. However, because the estimated values are at an acceptable level, this is tolerable. Thus, the proposed method has adequately robust performance 

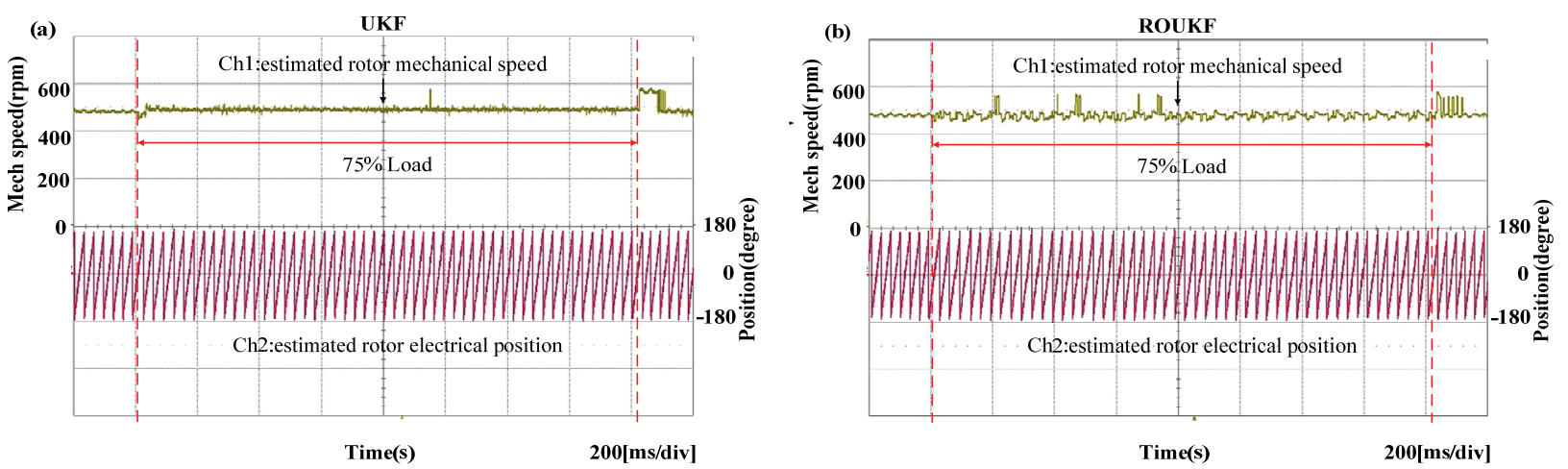

Fig. 6. Behavior and comparison of load test (500 rpm, $0 \rightarrow 75 \rightarrow 0 \%$ of rated load): (a) full-order form; (b) reduced-order form
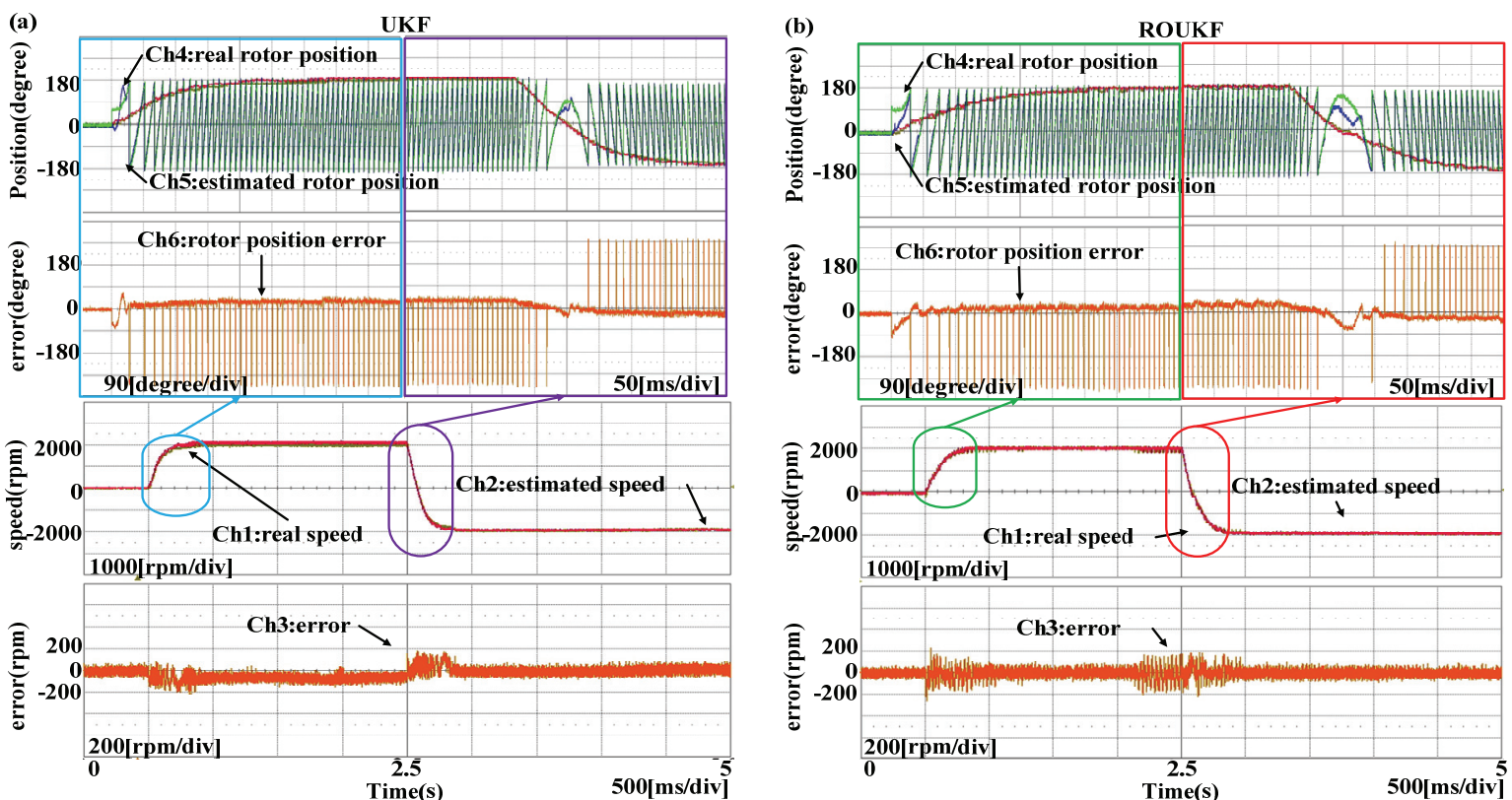

Fig. 7. Experimental waveforms from the detuned-parameter test $(2,000 \rightarrow-2,000 \mathrm{rpm}, 90 \%$ of stator resistance and inductance values): (a) full-order form, (b) reduced-order form

when experiencing a disturbance such as a load.

Fig. 7 presents the influence of the motor detuning parameters on the experimental waveform. The experimental conditions are set as $90 \%$ of the stator inductance and resistance values when the motor runs with forward and reverse rotation from 2,000 to $-2,000 \mathrm{rpm}$. In the case of $90 \%$ detuning parameter values, the performance of this method is similar to the full-order form with UT, but one has a slight oscillation in certain sections. It can be seen that the response speed is relatively slow according to the reduced-order form. This indicates that the proposed method has excellent performance regardless of the wrong parameter values.

The computation time under the same experimental conditions was compared. The computation time and code size for each form are listed in Table 1. In the case of the full-order form, there are slight differences between the
Table 1. Comparison of computational times

\begin{tabular}{c|c||c|c|c}
\hline \multicolumn{2}{c|}{ Type } & $\begin{array}{c}\text { Size of state } \\
\text { matrix }\end{array}$ & $\begin{array}{c}\text { Code Size } \\
\text { (byte) }\end{array}$ & $\begin{array}{c}\text { Computation } \\
\text { time }(\mu \mathrm{s})\end{array}$ \\
\hline \hline \multirow{2}{*}{ Basic } & Full-order & $4 \times 1$ & 11,627 & 75.34 \\
\cline { 2 - 5 } & Reduced-order & $3 \times 1$ & 8,529 & 49.61 \\
\hline \multirow{2}{*}{ Scaled } & full-order & $4 \times 1$ & 12,219 & 88.26 \\
\cline { 2 - 5 } & Reduced-order & $3 \times 1$ & 9,191 & 55.44 \\
\hline
\end{tabular}

scaled UT and basic UT. The presence or absence of the zeroth sigma point had an important effect on the computation time, which is reduced by $\sim 13 \mu$ s. The reducedorder form can reduce the computation time by $26 \mu \mathrm{s}$ (basic UT) and $33 \mu$ s (scaled UT). Thus, this method can reduce the computation time by $\sim 30 \mu$ s regardless of the UT type, while maintaining constant performance.

This paper shows how to reduce the code size and computation time of the Kalman filter. When a Kalman 
filter is used to estimate state values, the computational load is the main drawback. We significantly resolved this problem. Furthermore, according to the experimental results, the proposed Kalman filter exhibited proper estimation performance comparable to that of the conventional Kalman filters.

\section{Conclusion}

This paper investigates the application, design, and implementation of a reduced-order unscented Kalman filter for the state estimation of a vector-control motor drive without a position sensor. The performance of the proposed method was investigated over a wide range of operations, such as forward and reverse rotations at low and high speed, detuning, and disturbance tests. Compared with the full-order form, the proposed scheme greatly reduces the computational time by approximately $34.15 \%$ and $37.19 \%$ for basic UT and scaled UT, respectively. The accuracy and performance of this method are not significantly different from those of the full-order form. Moreover, we studied how the scaling parameters determine the type of UT. The experimental results demonstrate the validity of the proposed algorithm for the sensorless speed control of the PMSM. In future research, we will explore the reduction of the speed error near the zero point.

\section{References}

[1] H. Lin, K-Y. Hwang and B-I. Kwon, "An Improved Flux Observer for Sensorless Permanent Magnet Synchronous Motor Drives with Parameter Identification," Journal of Electrical Engineering \& Technology, vol. 8, no. 3, pp. 516-523, May 2013.

[2] H-W. Sim, J-S. Lee and K-B. Lee, "On-line parameter estimation of interior permanent magnet synchronous motor using an extended Kalman filter," Journal of Electrical Engineering \& Technology, vol. 9, no. 2, pp. 600-608, March 2014.

[3] S. J. Julier, J. K. Uhlmann and H. F. Durrant-Whyte, "A new approach for filtering nonlinear systems," in Proc. American Control Conf., Seattle WA, YSA, 1995, pp. 1628-1632.

[4] S. J. Julier, "The scaled unscented transformation," in Proc. American Control Conf. 2002, vol. 6, 2002, pp 4555-4559.

[5] Y. H. Kim "High Performance IPMSM Drives without Rotational Position Sensors Using ReducedOrder EKF," IEEE Trans. Energy Conversion, vol. 14, no. 4, pp. 868-873, 1999.

[6] M. C. Huang, A. J. Moses and F. Anayi, X. G. Yao, "Reduced-Order Linear Kalman Filter (RLKF) Theory in Application of Sensorless Control for Permanent
Magnet Synchronous Motor(PMSM)," in Conf. Rec. IEEE-ICIEA, 2006, pp. 1-6.

[7] J.-S. Jang, B.-G. Park, T.-S. Kim, D. M. Lee and D.-S. Hyun, "Parallel reduced-order extended Kalman filter for PMSM sensorless drives," in Proc. IEEE Ind. Electron. Soc. Annu. Conf., Nov. 2008, pp. 1326-1331.

[8] V. Smidl and Z. Peroutka, "Reduced-order squareroot EKF for sensorless control of PMSM drives," in Proc. IEEE Ind. Electron. Soc. Annu. Conf., Nov. 2011, pp. 2000-2005.

[9] M. Grewal and A. Andrews, Kalman Filtering: Theory and Practice Using MATLAB, 3nd Edition, Wiley-IEEE Press, 2008.

[10] Cheol Moon and Young Ahn Kwon, "Sensorless Speed Control of Permanent Magnet Synchronous Motor by Unscented Kalman Filter using Various Scaling Parameters," Journal of Electrical Engineering \& Technology, vol. 11, no. 2, pp. 237-252, Mar, 2016

[11] S. Jafarzadeh, C. Lascu, and M. Fadali, "State estimation of induction motor drives using the unscented Kalman filter," IEEE Trans. Ind. Electron., vol. 59, no. 11. pp. 4207-4216., Nov. 2012.

[12] T.-F. Chna, P. Borsje, and W. Wang "Application of unscented Kalman filter to sensorless permanentmagnet synchronous motor drive," in IEEE Int. Conf. Electr. Machines Drives, Miami, FL, 2009, pp. 631638.

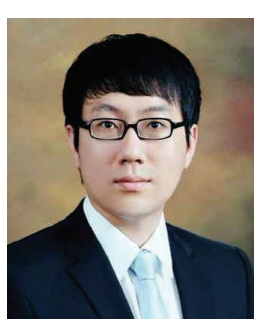

Cheol Moon received B.S and M.S degree in electrical engineering from Pusan National University, Busan, Korea in 2010 and 2012, respectively. $\mathrm{He}$ is currently working toward the Ph.D. degree.

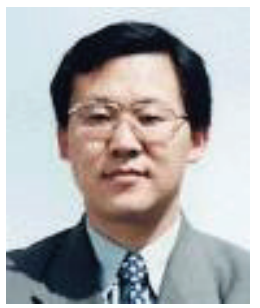

Young-Ahn Kwon received B.S, M.S, and $\mathrm{Ph} . \mathrm{D}$. degree in electrical engineering from Seoul National University, Seoul, Korea in 1978, 1983, and 1986, respectively. He is currently a Professor in the Department of Electrical and Computer Engineering, Pusan National University, Busan, Korea 